\title{
Field Testing of Lake Water Chemistry with a Portable and an AUV-based Mass Spectrometer
}

\author{
Harry F. Hemond, ${ }^{\mathrm{a}}$ Amy V. Mueller, ${ }^{\mathrm{a}}$ and Michael Hemond ${ }^{\mathrm{b}}$ \\ ${ }^{a}$ Department of Civil and Environmental Engineering, Massachusetts Institute of Technology, Cambridge, \\ Massachusetts, USA \\ ${ }^{b}$ Harvard-MIT Division of Health Sciences and Technology, Department of Biological Chemistry and \\ Molecular Pharmacology, Harvard Medical School, Boston, Massachusetts, USA
}

Two mass spectrometers (MS) are tested for the measurement of volatile substances, such as hydrocarbons and metabolic gases, in natural waters. KOALA is a backpackable MS operated from above the water surface, in which samples are pumped through a flow cell using a syringe. NEREUS is an underwater instrument hosted by an autonomous underwater vehicle (AUV) that is linked to a communications network to provide chemical data in real time. The mass analyzers of the two MS are nearly identical cycloids, and both use flat-plate membrane inlets. Testing took place in an eutrophic, thermally stratified lake exhibiting steep chemical gradients and significant levels of methane. KOALA provided rapid multispecies analysis of dissolved gases, with a detection limit for methane of $0.1 \mathrm{ppm}$ (readily extendable to $0.01 \mathrm{ppm}$ ) and savings of time of at least a factor of 10 compared to that of conventional analysis. The AUV-mounted NEREUS additionally provided rapid spatial coverage and the capability of performing chemical surveys autonomously. Tests demonstrated the need for temperature control of a membrane inlet when steep thermal gradients are present in a water body, as well as the benefits of co-locating all sensors on the AUV to avoid interference from chemically different waters entering and draining from the free-flooding outer hull. The ability to measure dissolved volatiles provided by MS offers potential for complementarity with ionic sensors in the study of natural waters, such as in the case of the carbonate system. (J Am Soc Mass Spectrom 2008, 19, 1403-1410) (C) 2008 American Society for Mass Spectrometry

$\mathrm{N}$ atural waters contain dissolved volatile substances of much practical or scientific interest, including metabolic gases such as oxygen, carbon dioxide, dinitrogen, nitrous and nitric oxides, hydrogen, and hydrogen sulfide; noble gases such as argon and neon; methane and various other light alkanes and alkenes; indicators of fuel such as BTEX (benzene, toluene, ethylbenzene, xylene) and chlorinated solvents such as TCE (trichloroethylene). Because of the large-volume, nonhomogeneity, and time-varying nature of many waters, spatiotemporally dense measurements of water chemistry may be needed. Moreover, many applications demand that there be little time delay between contacting a water volume and obtaining the requested data. However, conventional water sampling and laboratory analysis often involve not only delay but also risks of contamination, analyte degradation, or unintended volatilization.

These problems motivate the need for instruments capable of making chemical measurements in water in

Address reprint requests to Prof. Harry Hemond, Massachusetts Institute of Technology, Department of Civil and Environmental Engineering, Cambridge, MA 02139. E-mail: hfhemond@mit.edu the field, in near-real time. Until the advent of fieldportable mass spectrometers (MS), successes with in situ measurement of volatile chemicals have been largely restricted to sensors for individual substances (e.g., polarographic and optode-based oxygen sensors). Mass spectrometry intrinsically possesses multispecies capability, however, and a number of in situ or on-site portable MS for environmental chemical measurement have now been described [1, 2]. These instruments differ, in many cases importantly, with respect to mass range of analytes targeted, configuration of mass analyzer, nature of sample introduction system, type of vacuum system, degree of autonomy provided by control and data systems, and electrical power needs.

We describe here environmental applications of the NEREUS underwater MS and its closely related predecessor KOALA, a backpack-portable on-site MS (Figure 1). These instruments have, at their core, nearly identical mass analyzers and data systems. KOALA may be transported to the field and presented with water samples (in shallow waters and sediments, a probe can also be inserted directly into the medium of interest). In such mode of operation a human operator is in immediate control. NEREUS is deployed aboard an autonomous underwater 


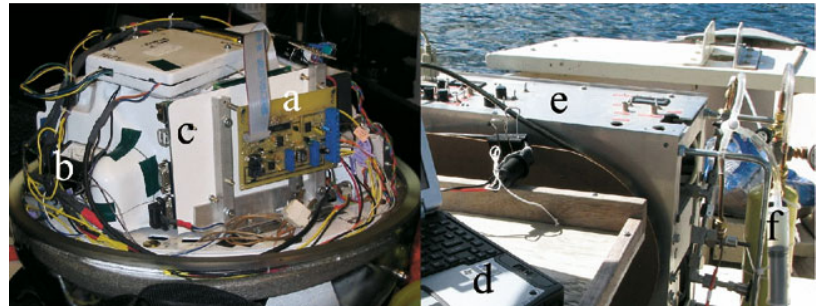

Figure 1. The NEREUS (left) and KOALA mass spectrometers. The upper half of the NEREUS pressure housing is removed to show layout: (a) safety monitoring circuitry, (b) ion pump current meter, and (c) back of TS-5500 computer mounting. Laptop (d) running Melite communicates with KOALA mass spectrometer (e), which is receiving samples pumped by syringe (f) through membrane inlet flow cell (see details in Figure 2).

robot, an advantageous mode of operation in large bodies of water. Such deployment requires that the instrument be waterproof and pressure-resistant, have a suitable interface with its host robot, be able to autonomously handle tasks such as calibration, respond appropriately to malfunctions, and not represent a serious risk to its host in the event of electrical faults or loss of buoyancy.

Although it is not within the scope of this paper to exhaustively review and compare the numerous possible configurations and comparative merits of different on-site and in situ mass spectrometers, we do discuss key considerations in the design of NEREUS and KOALA. We describe field experience with both instruments, demonstrating the utility of on-site mass spectrometry and in situ underwater mass spectrometry for practical chemical testing.

\section{Experimental}

\section{Mass Analyzer}

Mass analyzers that have been used successfully in portable applications include, but are not limited to, quadrupoles, magnetic analyzers in various geometries (including cycloids), and ion traps. A cycloid [3] has the advantages of being both velocity and direction focusing, of requiring no RF generator, and, except for the filament of the electron source, of requiring only modest (not exceeding a few hundred volts) DC voltages at low current.

Both NEREUS and KOALA use the same design of mass analyzer that is used in the CEC 21-620 laboratory mass spectrometer, a general-purpose instrument using a cycloidal analyzer of 1-inch pitch, formerly marketed by Consolidated Electrodynamics Corp. [4]. One key to enabling the use of this instrument's mass analyzer in portable operation is replacement of the original alnico magnet, which weighs about 80 pounds, with a very compact neodymium-iron unit. Details of the mass analyzer characteristics are described in Robinson and Hall [5], whereas general mechanical arrangements of NEREUS and KOALA mass spectrometers themselves are described in various publications by Hemond and
Camilli [6-8]; however, the instruments used in the present study have entirely reworked data systems, redesigned electrometers, addition of (or, in the case of NEREUS, change to) a Technologic Systems (Fountain Hills, AZ, USA) TS-5500 microcomputer for control and data acquisition, addition of electrical filtering to reduce noise, and redesigned inlet systems.

In the case of NEREUS, additional circuitry has also been provided for remote control of power as well as monitoring of internal battery voltage, ion pump current (which is also a measure of vacuum quality in the mass analyzer), and power to the ion pump. Provision is also made for various safety behaviors (see following text).

\section{Inlet System}

A membrane inlet is used for NEREUS and KOALA, both for simplicity and to enrich analyte relative to the water background. Simple membrane inlets forfeit the preseparation provided by a gas chromatograph (GC)based inlet, but avoid the cost and complication of mechanisms needed for discrete injection of samples, as well as the time delays required for GC separations. The inlets have a polymer membrane supported over a plate of stainless steel, having a diameter of $12 \mathrm{~mm}$, in which 220 holes of $0.089 \mathrm{~mm}$ radius are formed by electrical discharge machining (EDM) (Figure 2). The effective membrane area is $0.8 \mathrm{~mm}^{2}$, and $0.025 \mathrm{~mm}$ thick membranes of silicone rubber, polyethylene, or polypropylene have been used. Although the ultimate pressure capability is not known, the inlet has been tested to 800 $\mathrm{kPa}$ without damage.

NEREUS exposes the inlet directly to environmental waters; a flexible stainless tube of $6.3 \mathrm{~mm}$ OD and about $60 \mathrm{~cm}$ length extends from the mass spectrometer to the outer hull of the AUV, and the membrane is exposed to the flow of water past the outer hull. KOALA uses an

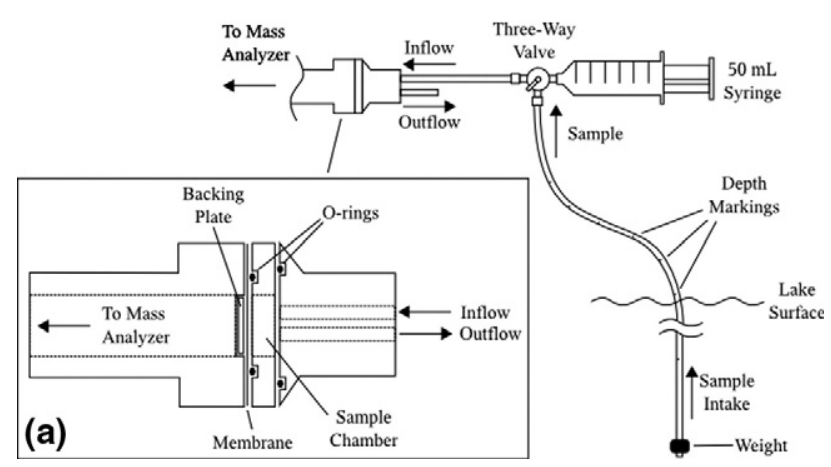

Figure 2. Schematic of membrane inlet/flow cell and of syringe system (see text). The syringe system is a very simple and convenient way of presenting water from desired depths to the membrane inlet of the KOALA instrument; a small motorized pump could fulfill the same function. In the case of NEREUS, the cap of the sample chamber (the component furthest to the right in inset a) is omitted, leaving the shallow sample chamber open and the membrane freely exposed to water flowing past the hull of the AUV. 
inlet identical to that used for NEREUS, with a flange attached over the face to form a $4 \mathrm{~cm}^{3}$ flow cell (Figure 2 ). Water is pulled from the environment and forced past the membrane using $3.1 \mathrm{~mm}$ ID nylon tubing of the necessary length (tens of meters) together with a $50 \mathrm{~mL}$ disposable syringe and a Luer three-way valve.

In the case of NEREUS, a complementary set of chemical sensors, embodied in a Hydrolab DataSonde model 4a (Hach Environmental, Loveland, CO, USA) water quality multiprobe, is attached midship on the port side of the hull and is connected to a serial port of the vehicle's computer via an Impulse MIL-9 cable. The Hydrolab probe includes a Clark electrode [9] to measure dissolved oxygen, thus providing an independent cross-check on one of the variables measured by NEREUS.

\section{Vacuum System}

NEREUS and KOALA use $8 \mathrm{~L} / \mathrm{s}$ sputter-ion pumps (Varian Co., Palo Alto, CA, USA), powered at $+3 \mathrm{kV}$ from a DC-DC power supply, with a $1000 \mu \mathrm{A}$ meter in series with the ground terminal to provide indication of current [6]. Although such pumps are not able to self-start from atmospheric pressure, their low power consumption is compatible with power-limited portable applications. On NEREUS only, ion pump current is also read via an ADC connected to the GPIO port on the TS-5500 microcomputer, as well as monitored by a hard-wired comparator circuit to provide emergency shutoff behaviors even when the computer is not running.

\section{Power}

Power (12 V DC) for both instruments is provided by an internal 4 amp-h sealed lead acid battery, providing over $1.5 \mathrm{~h}$ of self-contained operation and facilitating continuity of power to the sputter ion pump; an external $12 \mathrm{~V}$ source is routinely connected to maintain battery charge as well as allow extended operating time. In the case of NEREUS, a dedicated DC-DC converter is provided on the AUV, in which the power is supplied via a 9-pin MIL-9 Impulse (San Diego, CA, USA) underwater connector, waterproof cabling, and corresponding watertight penetrations that connect NEREUS to the vehicle control sphere. Power is controlled internally via a relay board; a watertight switch, accessible from the top outside of the AUV while it is in the water, provides positive control independent of the state of the vehicle or mass spectrometer computers.

Twelve volt power is converted to the needs of individual circuits via modular DC-DC converters. The mitigation of electrical noise is an important consideration in this situation, given that such converters frequently have several 10s of millivolts of ripple associated with their outputs. In addition, the environment of the AUV is generally electrically noisy because of the widespread use of switching circuitry, especially that associated with the relatively high power levels of the propulsion and control surface motors. Because this noise tends to became coupled into the mass spectrometer circuitry, the most noise-critical circuits receive their power via LC filters. Typically, LC filters using inductors of several millihenrys and $100 \mu \mathrm{F}$ capacitors are satisfactory; it is often necessary to include an inductive element on the ground return line as well as in the + and - supply lines to fully suppress commonmode noise.

\section{Autonomous Underwater Vehicle (AUV)}

The NEREUS mass spectrometer has been deployed aboard an Odyssey II class AUV [10,11]. The Odyssey II has a displacement of about $190 \mathrm{~kg}$ and overall length of about $2 \mathrm{~m}$. The AUV consists of a streamlined outer, free-flooding hull, and two pressureresistant spheres that carry vehicle control electronics and an instrument payload, respectively. Power is provided by a $2 \mathrm{~kW}$-h pressure-compensated Li-polymer battery (Bluefin, Cambridge, MA, USA), and the motors for control surface operation and propulsion are also oilfilled for pressure compensation. The vehicle has fixed buoyancy, and is normally trimmed to be about $20 \mathrm{Nt}$ positive; depth is thus maintained by hydrodynamic body forces on the hull, and depth adjustments are effected by controlling vehicle pitch.

\section{Safety Measures}

Certain risks are increased by operation of an MS aboard an AUV. One is loss of buoyancy attributed to membrane rupture, potentially leading to loss of the vehicle and instrument. Thus provision is made on NEREUS for a solenoid valve to isolate the MS in event of vacuum loss; the Odyssey II can also release a $2 \mathrm{~kg}$ drop weight to provide emergency buoyancy. The NEREUS can provide advance warning of leaks in its waterproof housing (which is normally evacuated to about $0.75 \mathrm{~atm}$ ), both via a mechanical gauge that is readable through the glass sphere, and via a pressure sensor interfaced to an ADC port on the TS-5500. To mitigate hazards of hydrogen from the battery, a catalytic battery gas recombining unit (Catylator, Hydrocap Corp., Miami FL, USA) is installed. Also for safety, the vehicle's $2 \mathrm{~kW}$-h battery is removed before charging under direct control of the manufacturer's software.

\section{Mechanical Interface to the AUV}

The NEREUS mass spectrometer is mounted in the forward sphere position of the Odyssey II vehicle. A rigid foam cover occupies the volume between the sphere and the upper half of the outer hull and provides buoyancy to compensate for the small negative buoyancy of the instrument itself $(\sim 30 \mathrm{Nt})$. To prevent adverse effects on vehicle trim and underwater stability, the NEREUS internal arrangement has a center of 
mass close to the center of volume of its pressure sphere. Final trimming of the vehicle is accomplished with small weights or pieces of buoyant foam added inside the outer hull. The vehicle is shown with the upper hull removed, and also in its field configuration, in Figure 3.

\section{Magnetic Compensation of AUV Compass}

One issue associated with the choice of a magnetic mass spectrometer is potential interference with the vehicle's magnetic compass. Although the MS is located nearly a meter from the compass contained in the attitude and heading reference system (AHRS) of the AUV, the fringing field of NEREUS' 0.4 Tesla magnet was found to introduce up to $50^{\circ}$ of compass error on some vehicle headings. Moreover, the error varied strongly and nonlinearly with heading.

Implementing an empirically determined compass error lookup table in the vehicle control software provided marginally satisfactory heading accuracy. A much better solution resulted from installation of a version of the AHRS that contains an internal algorithm for compass correction, after which vehicle heading error could not reliably be discerned from zero using a good-quality handheld bearing compass (Suunto KB-14).

\section{Control and Data Handling Software}

The control and data handling requirements vary greatly between NEREUS and KOALA. Both to facili-

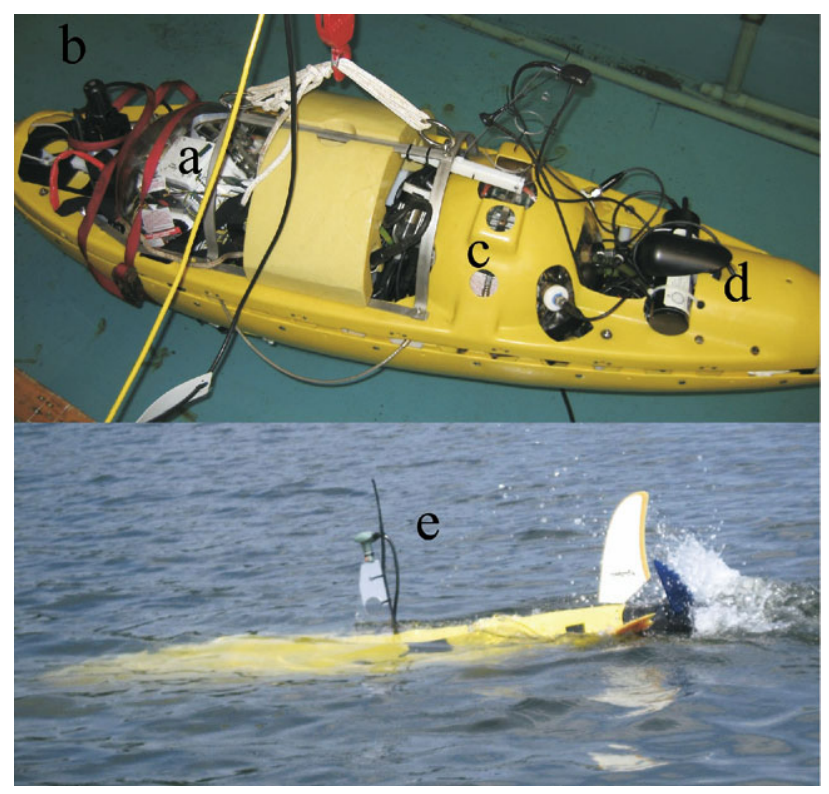

Figure 3. The Odyssey II host vehicle showing, in top panel: (a) NEREUS spherical housing in the bow, (b) acoustic transducer for underwater communication, (c) location of vehicle control electronics (with inner hull covering in place), and (d) emergency transponder. Bottom panel shows vehicle beginning mission; mast (e) carries GPS unit and communications antenna. tate software development and to maximize commonality between NEREUS and the manually operated KOALA instrument, the same control program (Iaira) is used to operate both machines. A flexible GUI (Melite) interacts with Iaira and provides a human operator interface, whereas iNereus provides an AUV interface. The AUV is normally operated as part of a control and data network, which also consists of moored buoys and a shore station, linked using both underwater acoustic communication and abovewater radio networking.

Acoustic/wireless network. The above-referenced network is described in detail in Hemond and colleagues [12]. Briefly, the network contains the AUV, several multirole buoys, and a "shore station" that can also be operated aboard a manned surface chase boat. Data are received, visualized, and logged, and commands can be issued, from the shore station. Each component is based on MOOS, a publish-and-subscribe operating system developed initially for robot control [13], for which several custom instrument-operating and communications processes have been developed.

Iaira. Iaira, the mass spectrometer control program, is implemented in Perl running on Linux on the TS-5500 microcomputer and controls a set of lowlevel drivers, written in $C$, that communicate directly with the NEREUS or KOALA mass spectrometer hardware. Iaira interacts with a human or robotic user via human-readable ASCII text strings and communicates with a low-level data acquisition board driver using ASCII strings sent via a pair of named Unix pipes.

In the normal mode of operation Iaira starts automatically. When given a command to read ion intensity at a desired $\mathrm{m} / \mathrm{z}$ value, Iaira calculates the DC voltage required to set the cycloidal mass filter to this $m / z$ value, and commands a digital-to-analog converter (DAC), whose output is amplified to a value between 0 and 300 $\mathrm{V}$ using a high-voltage op-amp (Apex PA-88). Voltage dividers provide appropriate voltages, each proportional to the op-amp output, to various electrodes in the mass filter and ion source. Iaira then reads ion current, averaging any specified number of read operations. Averaging is carried out internally by the low-level driver to minimize communications overhead. The driver also inserts a delay (typically $400 \mu$ s per volt change of DAC is used) before reading ion current, thus minimizing artifacts from capacitive coupling of voltage transients into the electrometer when large changes in $\mathrm{m} / \mathrm{z}$ are made quickly.

Calculation of the DC voltage needed to set the mass filter to a given $\mathrm{m} / \mathrm{z}$ follows the relationship:

$$
\text { Voltage }=\text { Slope } /(m / z)+\text { Offset }
$$

The Offset parameter removes any DC offset in the hardware, although in practice offset has been found to be less than $1 \mathrm{mV}$. Slope and Offset may be set manually but can also be set by Iaira using an automatic two- 
point calibration with the Cal Auto command. Two peaks (user-selectable-in practice $m / z 18$ and $m / z 28$ are good choices) are read and smoothed, and the apparent $\mathrm{m} / \mathrm{z}$ values at the center of each peak are then used to compute updated Offset and Slope values.

Iaira also reads from the built-in 12-bit ADC and the GPIO (general purpose input-output) hardware of the TS-5500 minicomputer, enabling it to monitor and report the internal pressure of the pressure sphere as well as power supply voltages. By raising a GPIO pin, it can also trigger a safety behavior, such as sending a signal to a solenoid valve in response to excessive ion pump current. Iaira computes an average of the last ten ion pump current readings and will trigger the safety behavior if the average value or the maximum value exceeds the respective threshold.

Iaira logs all acquired data to a Linux ext2 filesystem on a compact flash medium; each log line includes a timestamp, $m / z$ value, current intensity, and the word "data" to facilitate logfile analysis. This allows recovery of data after a mission even if communications are disrupted. Other information, such as hardware status and debugging messages, can be logged in a configurable manner. The possibility of filesystem corruption if the system loses power or is shut down improperly is mitigated by mounting the filesystem in synchronous mode so that write operations are executed immediately instead of being cached and potentially leaving the filesystem in an invalid state. Another potential cause of data corruption-the eventual failure of the compact flash medium itself after repeated write operations-is unavoidable but has not proved to be a problem to date.

Melite. Although NEREUS and KOALA can be operated manually using a terminal program such as Minicom, we developed a graphical program, Melite, that provides a far more convenient and informative user interface. Melite communicates with Iaira over a serial line and is implemented in Perl on Ubuntu Linux 6.06 LTS using the Perl/Tk graphical widget set. Spectra are rendered using gnuplot and the Chart::Plot module, obtained from the CPAN archive at www.cpan.org.

Melite supports the full set of Iaira commands and also provides additional high-level functions: (1) multiple scan ranges over various peaks of interest can be plotted on the same graph; (2) multiple graphs can be shown; (3) graphs and/or data can be saved for later use; (4) Melite can be configured to execute scans periodically; (5) calibration and hardware status information are continuously presented in the graphical interface; and (6) $\mathrm{m} / \mathrm{z}$ values for two-point calibration can be user-specified via the graphical interface. Arbitrary commands may also be passed directly to Iaira.

iNereus. iNereus runs on the AUV computer and acts as the main control and data pathway between Iaira and the aforementioned MOOS community on the AUV. The main functions of iNereus are as follows: (1) formulate data requests to be sent to Iaira according to scheduled requests or real-time user requests from the MOOS system; (2) process returned spectrum information; and (3) post data to the MOOS database for logging and, if desired, transfer to the shore station via radio (when the AUV is surfaced) or via an underwater acoustic network.

Like Melite, iNereus communicates with Iaira over an RS-232 serial line using Iaira's standard set of ASCII commands. Connection from the AUV's computer is made via three conductors of the aforementioned watertight cable between NEREUS and the vehicle control sphere. iNereus implements an automated user interface for all Iaira functions including emission regulator control, remote shutdown, system monitoring, manual/ automatic calibration, full and partial spectrum scans, single $m / z$ value scans, and scan averaging. A list of chemical species, including the expected $m / z$ value for the species and an acceptable window over which to scan for this $m / z$ value (this window may be made as narrow as a single point), is specified in the AUV's Mission file during mission setup. iNereus assigns the maximum response in the specified $\mathrm{m} / \mathrm{z}$ range as the corresponding output value. This methodology allows iNereus to account for small amounts of calibration drift as might occur during long missions or missions spanning wide temperature ranges; periodic automatic $m / z$ calibration can also be used toward the same end.

Because scan and calibration requests can be both periodic and on-demand, iNereus implements queue management for event scheduling. Prescheduled events are executed in the order in which their timers expire. Because Iaira has no provision for interrupting an ongoing scan, real-time requests are added to the queue immediately after completion of any event in progress.

Streaming input from Iaira is parsed to retrieve both calibration data from a calibration-display string and scan data from a list of coupled $\mathrm{m} / \mathrm{z}$ and voltage values. All data are logged aboard the AUV; in addition, current calibration, system monitoring parameters, and data for up to ten chemical species of interest can be transferred via acoustic modem in real time, such as for mission monitoring. Additional details concerning underwater data transmission are provided in Hemond et al. [12].

\section{Field Methods}

KOALA and NEREUS have been operated in a variety of laboratory and field settings. Data presented here are from Upper Mystic Lake (UML), located north of Boston, MA. This eutrophic urban lake has been the subject of several geochemical studies [e.g., 14-16] and experiences strong thermal stratification in summer, at which time strong chemical gradients develop across the thermocline (the depth of maximum temperature gradient). Data presented here were obtained in 2007 during stratified conditions. 
Surface deployment using syringe-pumping of water from depth. KOALA was deployed aboard a small boat at UML (Figure 1). For sampling, $3.2 \mathrm{~mm}(1 / 8$ inch) ID tubing was marked at regular intervals to provide depth reference, weighted at the open end, and lowered to desired depths in the lake (Figure 2). The syringe was cycled seven times, flushing the sample line by threefold its volume, each time expelling the water through the flow cell containing the membrane inlet. Melite was used to generate local scans of $\pm 0.5 \mathrm{~m} / z$ about the peaks for methane, oxygen, nitrogen, and carbon dioxide. MS trap current was set at $10 \mu \mathrm{A}$.

Field testing of NEREUS aboard the Odyssey II. The NEREUS/Odyssey II vehicle is transported to field sites on a custom launching cart, the mass spectrometer being provided with external $12 \mathrm{~V}$ power to avoid discharge of its internal battery during transport. The AUV is powered up for prelaunch checks, at which time the vehicle also takes over the electrical load of the mass spectrometer. The cart is manually rolled into the water for launching.

Typically the vehicle is first run on a series of test missions to verify its correct operation; the mass spectrometer is then activated using the switch on top of the AUV hull. The instrument has been typically run at the very low trap current of $5 \mu \mathrm{A}$. Initial qualitative $(\mathrm{m} / \mathrm{z})$ calibration of the MS is carried out manually using the shore station computer to communicate with Iaira via the terminal program Minicom, which is installed on the AUV computer and temporarily bypasses the iNereus process. Initial values of Slope and Offset (eq 1) are entered, typically being given their most recent values from a previous operation. A manually commanded scan is visually inspected to verify that the peaks chosen for calibration (typically the aforementioned peaks of dinitrogen at $\mathrm{m} / \mathrm{z} 28$ and water at $\mathrm{m} / \mathrm{z}$ 18) fall within the adjustable-width windows that are examined by the autocalibrate function, Cal Auto. Initial Slope and Offset can be changed as needed until the peaks chosen for calibration fall within a specified $\mathrm{m} / \mathrm{z}$ range (typically \pm 0.4 ) of their true values, although in practice such iteration is normally not necessary. Cal Auto is subsequently run to automatically recalculate and apply new Slope and Offset values, resulting in accurate instrument calibration. Finally, the iNereus process is started, after which the MS operates autonomously according to instructions coded into the iNereus configuration file. Typically this involves local scans for methane, oxygen, and nitrogen, as well as scans across $\mathrm{m} / \mathrm{z}$ values where peaks are not expected to be present, to establish baselines.

\section{Results and Discussion}

\section{Upper Mystic Lake, Observations from Surface}

Dissolved gas data from four different depths on September 12 show features expected in an eutrophic stratified lake (Figure 4). Oxygen levels decreased with
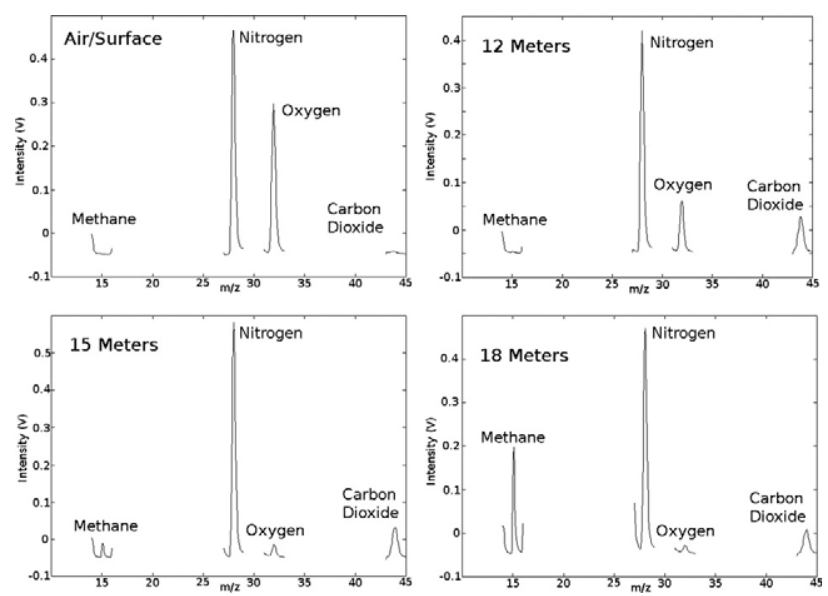

Figure 4. Chemical data from UML as measured by KOALA using experimental setup of Figure 1. Steep vertical gradients of methane, carbon dioxide, and oxygen are evident in panels for 12 , 15 , and $18 \mathrm{~m}$ depth, respectively (see text).

depth, becoming nearly zero below the thermocline, as a result of consumption by lake biota. Nitrogen levels are lowest near the surface, as expected, because of the decrease in nitrogen solubility and consequent loss of dinitrogen to the atmosphere as surface waters warm up during the summer. The carbon dioxide signal increases with increased depth, rising sharply below the thermocline, primarily a result of biotic carbon dioxide production via both oxygen-consuming respiration and a variety of anaerobic processes [14]. A substantial methane signal (measured at $\mathrm{m} / \mathrm{z} 15$ to avoid the need to correct for doubly charged oxygen) is evident in the deeper water, a result of organic matter mineralization in an organic-rich and strongly oxygen depleted environment, such as the bottom sediments of the lake.

The electrometer outputs presented earlier are related to chemical concentrations by calibration factors, which may be estimated from gas concentrations measured by conventional methods on dates close to Sept 12 (C. Varadharajan, personal communication). For an inlet membrane at $20^{\circ} \mathrm{C}$ and with 10 microamperes of MS trap current, methane concentration in micromoles per liter equaled $1.8 \times$ electrometer output in millivolts. Given electrometer noise of the order of $1-2 \mathrm{mV}$, the corresponding detection limit for methane is of the order of $5 \mu \mathrm{mol} / \mathrm{L}$, or 0.1 parts per million. For nitrogen and oxygen the calibration factors were approximately 1.1 and $3 \mu \mathrm{mol} \mathrm{L}^{-1} \mathrm{mV}^{-1}$, respectively. The detection limit can be lowered by increasing trap current and by increased signal averaging. Also, trap current can be increased approximately fivefold, increasing the signal proportionally with no change in noise level. The data reported here are with fivefold signal averaging; increasing this tenfold results in a baseline noise decrease of about threefold (i.e., varying inversely as the square root of number of reads per datum). 


\section{Chemical Profiles Observed Using NEREUS/Odyssey II}

A test mission of NEREUS aboard the Odyssey II AUV in UML is illustrated in Figures 5-7. Figure 5 illustrates a dive profile in which the vehicle traversed the lake basin from southeast to northwest, diving to and leveling out at a depth of $18 \mathrm{~m}$, then terminating the powered mission and floating passively to the surface. At $580 \mathrm{~s}$, the $x$ - and $y$-coordinates appear discontinuous, at the time the vehicle reacquires a GPS fix. This is a measure of accumulated navigational error when the vehicle was underwater, navigating by dead reckoning, and illustrates the shortcomings of the manually generated lookup table to correct for compass error. Underwater navigation error with the new AHRS that was subsequently installed, and which has a built-in compasscorrection algorithm, is only a few meters over such a course (data not shown).

The oxygen signals from NEREUS and from the Hydrolab multiprobe are essentially in agreement (Figure 6). They also agree with the surface-based measurements of Figure 4; note that $200 \mathrm{mV}$ is the electrometer baseline. The small local maximum in the multiprobe data is hypothesized to be an artifact of time lag in a temperature-compensation circuit. However, it is not possible to rule out the existence of a true local maximum of dissolved oxygen. This is possible because the Hydrolab and the NEREUS inlet are separated by over a meter and are on opposite sides of the vehicle. Alternatively, a very localized oxygen maximum could have been observed by the multiprobe but not the mass spectrometer because of the much higher frequency of
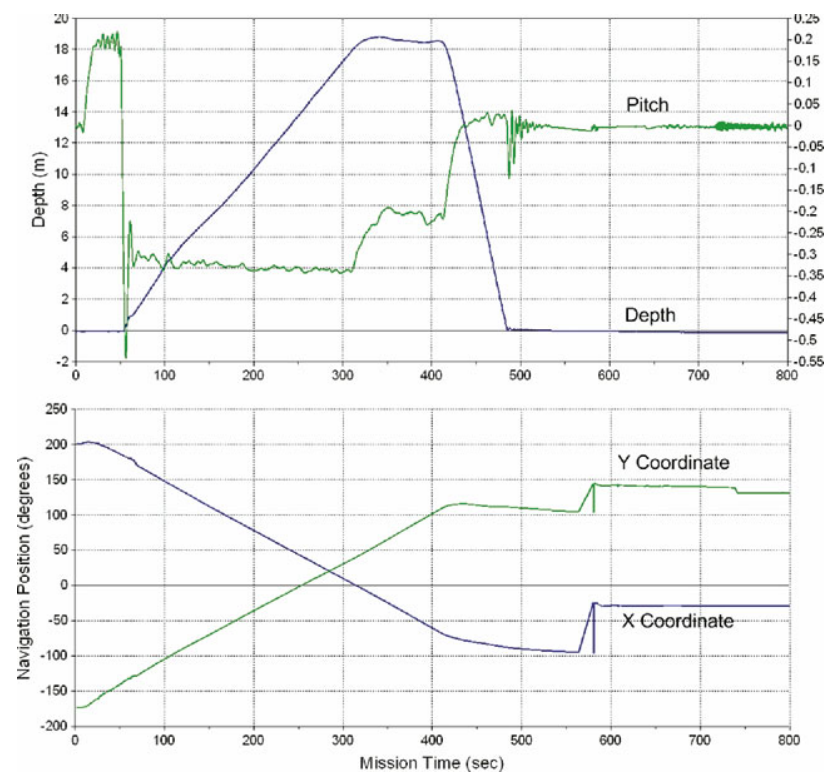

Figure 5. Physical mission profile in UML; corresponding chemical data are shown in Figures 6 and 7. Vehicle dives to, and levels out at, $18 \mathrm{~m}$ depth while traveling a southeast to northwest path across the lake. Mission terminates at time-out, and the AUV floats passively to the surface (see text).

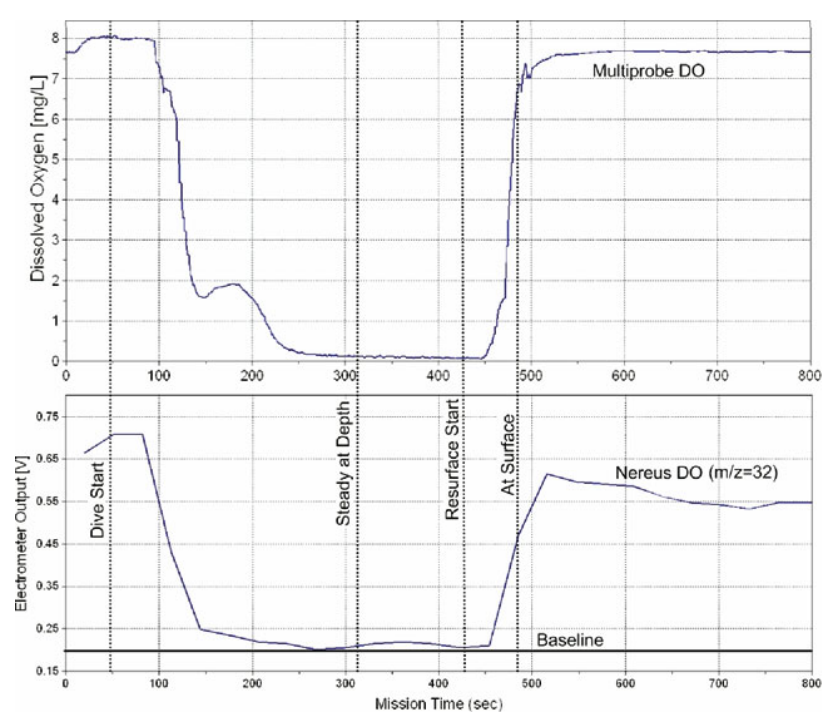

Figure 6. NEREUS oxygen measurement and Hydrolab oxygen measurements during the mission shown in Fig. 5 (see text).

the multiprobe readings (although the intrinsic time response of its membrane inlet is about $10 \mathrm{~s}$, mass spectrometer oxygen scans were reported only at 30-s intervals, whereas multiprobe readings were reported approximately every second). It is also noted that the dissolved oxygen signal does not return at once to its premission value when the vehicle surfaces, a predictable artifact of the AUV's free-flooding hull, which becomes filled with cold anoxic water during the traverse of the lake. When the vehicle floats to the surface, cold and dense anoxic water accumulated at depth can drain from the outer hull and flow past the membrane inlet, thus influencing the chemical signature. This is not a factor while the AUV is moving under power.

Dissolved nitrogen data are shown in Figure 7. This plot illustrates the temperature sensitivity of the unheated membrane inlet; although the nitrogen signal appears to decrease with depth, the actual concentration is expected to be somewhat higher at greater depth, as is confirmed in the KOALA data in Figure 4.

The methane signal in Figure 7 peaks at $100 \mathrm{mV}$, corresponding to $220 \mu \mathrm{mol} / \mathrm{L}$ using the calibration

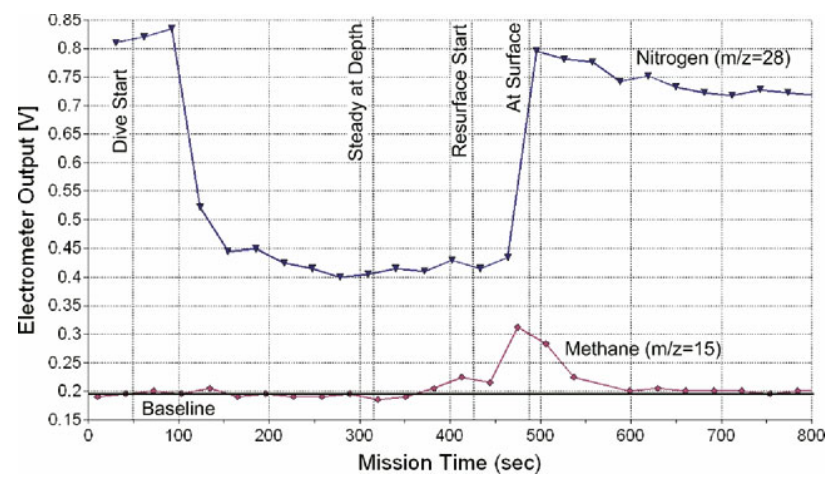

Figure 7. Profiles of dinitrogen and methane signals during mission shown in Figure 5 (see text). 
factor derived from the near-identical KOALA instrument at the temperature of the lake surface (correcting for different trap currents). This is almost certainly an underestimate resulting from the sensitivity of membrane permeability to the cold bottom water. The time lag is also consistent with lowered membrane permeability associated with the low water temperature at depth. A methane signal continues to be seen for at least a minute after the vehicle surfaces, reflecting the drainage of lake bottom water from the outer hull when the vehicle's propulsion is shut off and the AUV floats passively after the dive into cold methane-rich water.

\section{Conclusions}

Field observations confirm both the feasibility and utility of measuring gases of interest at ambient levels in natural waters using on-site or in situ mass spectrometry. Manual sampling works well and is much faster (at least 10-fold) than conventional sample collection, transport, and in-lab analysis. Among the merits of manual on-site operation are simplicity and the fact that water samples, which are brought to the surface through a small-diameter tube, all tend to be at the surface temperature of the lake when analyzed.

Per unit of time expended, use of the robotic platform provided greatly increased spatial coverage compared to that of surface-based sampling. The AUV avoids the time-consuming processes of lowering and fully flushing the sample line before measurement, while also providing location and time stamping of each datum. Measurement in situ also avoids possible degassing when samples are brought to the surface. Another incidental advantage is the avoidance of surface boat traffic in heavily utilized water bodies. Disadvantages include the increased complexity of a robotic system and the additional effort required to deploy and maintain an AUV. The optimum choice depends on a weighing of costs and advantages on a case-by-case basis.

The data indicate the need for membrane temperature control at sites where the inlet is exposed to very low or rapidly changing temperatures, as when the AUV occupies different portions of a strongly thermally stratified water environment such as UML. In addition, although the sensitivity of the mass spectrometer appears stable from day to day, except in response to temperature changes, a rigorous and more frequent quantitative calibration procedure will prove essential for many missions. The issue of water drainage from a free-flooding outer hull does not appear to be a problem for a vehicle running under cruise power, but is of concern where it is desired to run an AUV platform at very low speeds-for example, to increase the spatial resolution of chemical measurements made from the vehicle. An example could be a high-resolution vertical chemical profile of the thermocline region of a lake, which could be gained by shutting off a robot's propulsion below the thermocline, then measuring water chemistry as the vehicle floats slowly upward. In such cases, it will be necessary to locate penetrations of the outer hull to avoid proximity to sensors.

\section{Acknowledgments}

This work was supported by NSF EAR 0330272, by a grant from BP, by MIT Sea Grant, by the W. Leonhard Chair, and by NIEHS P30 ES002109. H. Nepf and C. Chryssostomidis are co-PIs on EAR 0330272 and provided valuable discussions; J. Morash, V. Polidoro, R. Damus, and J. Eskesen took part in vehicle construction and field testing, and D. Southern piloted several AUV missions.

\section{References}

1. Kotiaho, T. On-Site Environmental and In-Situ Process Analysis by Mass Spectrometry. J. Mass Spectrom. 1995, 31, 1-15.

2. Richardson, S. Mass Spectrometry in Environmental Sciences. Chem. Rev. 2001, 101, 211-254

3. Bleakney, W.; Hipple, J. A. A New Mass Spectrometer with Improved Focusing Properties. Phys. Rev. 1938, 13, 521-529.

4. Willard, H. H.; Merritt, L. L.; Dean, J. A. Instrumental Methods of Analysis, 3rd edition; D. Van Nostrand Co.: Princeton NJ, 1958.

5. Robinson, C. F.; Hall, L. G. A Small General Purpose Cycloidal-Focusing Mass Spectrometer. Rev. Sci. Instrum. 1956, 27, 504-508.

6. Hemond, H. F. A Backpack-portable Mass Spectrometer for Measurement of Volatile Compounds in the Environment. Rev. Sci. Instrum. 1991, 62, 1420-1425.

7. Hemond, H. F.; Camilli, R. NEREUS: Engineering Concept for an Underwater Mass Spectrometer. Trends Anal. Chem. 2002, 21, 526-533.

8. Camilli, R.; Hemond, H. F. NEREUS/Kemonaut, a Mobile Autonomous Underwater Mass Spectrometer. Trends Anal. Chem. 2004, 23, 307-313.

9. Hitchman, M. L. Measurement of Dissolved Oxygen; John Wiley \& Sons/Orbisphere Laboratories: Geneva, 1978.

10. Bellingham, J. G.; Goudey, C. A.; Consi, T. R.; Bales, J. W.; Atwood, D. K.; Leonard, J. J.; Chryssostomidis, C. A Second Generation Survey AUV. In Proceedings of the 1994 Symposium on Autonomous Underwater Vehicle Technology; Oceanic Engineering Society of IEEE: Cambridge, MA, July 1994; pp 148-155.

11. AUV Laboratory at MIT Sea Grant. http://auvlab.mit.edu/vehicles.

12. Hemond, H.; Cheung, J.; Mueller, A.; Wong, J.; Hemond, M.; Mueller, D.; Eskesen, J. The NEREUS In-Lake Wireless/Acoustic Chemical Data Network. Limnol. Oceanogr. Methods 2008 (in press).

13. Newman, P. MOOS-A Mission-oriented Operating Suite. Massachusetts Institute of Technology, Department of Ocean Engineering, Technical Report, 2002. This document is archived and available on-line at http://ora.ouls.ox.ac.uk:8081/10030/1773.

14. Senn, D. B.; Hemond, H. F. Nitrate Controls on Iron and Arsenic in an Urban Lake. Science 2002, 296, 2373-2375.

15. Rauch, S.; Hemond, H. F.; Brabander, D. J. High Spatial Resolution Analysis of Lake Sediment Cores by Laser Ablation-Inductively Coupled Plasma-Mass Spectrometry (LA-ICP-MS). Limnol. Oceanogr. Methods 2006, 4, 268-274.

16. Spliethoff, H. M.; Hemond, H. F. History of Toxic Metal Discharge to Surface Waters of the Aberjona Watershed. Environ. Sci. Technol. 1996, $30,121-128$. 